\title{
The efficacy and safety of bupropion sustained- release formulation for the treatment of major depressive disorder: a multi-center, randomized, double-blind, placebo-controlled study in Asian patients
}

This article was published in the following Dove Press journal:

Neuropsychiatric Disease and Treatment

27 August 2013

Number of times this article has been viewed

\author{
Yoshifumi Koshino' \\ Won-Myong Bahk ${ }^{2}$ \\ Hideaki Sakai ${ }^{3}$ \\ Takayuki Kobayashi ${ }^{4}$ \\ 'Iris Medical Clinic, Kanazawa \\ University, Ishikawa, Japan; ${ }^{2}$ Yeouido \\ St Mary's Hospital, The Catholic \\ University of Korea, Seoul, South \\ Korea; ${ }^{3}$ Meguro Station East \\ Mental Clinic, Shinagawa, Tokyo, \\ Japan; ${ }^{4}$ Medicines Development \\ (Neurosciences), Development and \\ Medical Affairs, GlaxoSmithKline, \\ Shibuya, Tokyo, Japan
}

\begin{abstract}
This study was conducted to compare the efficacy and safety of bupropion sustainedrelease (SR) formulation orally administered at daily doses of $150 \mathrm{mg} /$ day (once daily) and $300 \mathrm{mg} /$ day (150 mg twice daily) for 8 weeks versus placebo in Asian patients with major depressive disorder. The mean change from baseline in Montgomery-Åsberg Depression Rating Scale (MADRS) total score at week 8 was compared between each of the bupropion SR dose groups and the placebo group using an analysis of covariance with the multiplicity adjustment by Dunnett's step-down procedure. A total of 569 subjects met all of the inclusion criteria and proceeded to the treatment phase. The subjects proceeding to the treatment phase included 454 Japanese patients and 115 Korean patients. There was no statistically significant difference between each of the bupropion SR dose groups and the placebo group in the primary efficacy endpoint of change from baseline in MADRS total score at week 8 . Similar results were generally obtained for all of the secondary efficacy endpoints. The secondary analysis and the other subgroup analysis did not show a statistically significant difference in efficacy. There was no substantial difference in the type, severity, and incidence of adverse events (AEs) between the bupropion SR dose groups and the placebo group, which indicates a favorable safety profile for bupropion SR. There were no significant findings in subjects treated with bupropion SR in regard to sexual dysfunction, weight change, and withdrawal syndrome, which are frequently recognized as clinical concerns associated with selective serotonin reuptake inhibitors, widely used for the treatment of depression.
\end{abstract}

Keywords: bupropion SR, placebo, major depressive disorder, Japan, Korea

\section{Introduction}

Bupropion hydrochloride (GlaxoSmithKline plc, Brentford, Middlesex, UK) is an antidepressant of the aminoketone class, chemically unrelated to tricyclic antidepressants (TCAs), tetracyclic antidepressants, selective serotonin reuptake inhibitors (SSRIs), serotonin noradrenaline reuptake inhibitors (SNRIs), or other known antidepressant agents. Bupropion is an inhibitor of dopamine and noradrenaline reuptake. Although bupropion is recognized as an important treatment option ${ }^{1-4}$ for the treatment of major depressive disorder (MDD), it is not yet available in Japan. However, bupropion is already available in South Korea, but no randomized placebo-controlled trial has been conducted in Korean patients with MDD.
Correspondence: Takayuki Kobayash 4-6-15, Sendagaya Shibuya-ku,

Tokyo, I5I-8566, Japan

Tel +8I 357865092

Fax $+8 \mid 357865223$

Email takayuki.2.kobayashi@gsk.com 
Bupropion was first approved in the USA in 1985 as Wellbutrin, a three times daily dosing formulation. Subsequently, Wellbutrin sustained release (SR), which is the investigational product of this study, was developed as a twice-daily SR formulation that can achieve an area under the concentration-time curve (AUC) comparable to that of Wellbutrin, with a lower maximum plasma concentration.

The primary objective of this study was to investigate the efficacy of oral bupropion SR at doses of $150 \mathrm{mg}$ /day (given as a single daily dose) and $300 \mathrm{mg} /$ day (given as $150 \mathrm{mg}$ twice daily) for 8 weeks compared with placebo, using the Montgomery-Åsberg Depression Rating Scale (MADRS) ${ }^{5}$ as an indicator, in a multicenter, randomized, double-blind, parallel-group study in Japanese and Korean patients with MDD. The dosage and administration used for this study were consistent with the approved dosage and administration for the treatment of MDD in the USA. This study was carried out as the first Asian collaboration study of bupropion patients with MDD in Japan and South Korea.

\section{Materials and methods}

After obtaining consent from the subject and his/her proxy consent (if the subject was aged $<20$ years at the time of giving consent), the investigator registered the anonymized subject number via a centralized registration and randomization system. Following confirmation of eligibility to participate in the study by investigators, subjects were randomly allocated into three groups: bupropion SR $150 \mathrm{mg} /$ day (BUP150), $300 \mathrm{mg} /$ day (BUP300), or placebo, in a 1:1:1 ratio at the start of the treatment phase (week 0) (ClinicalTrials.gov identifier: NCT01138007). Investigational products were administered twice daily, in the morning and in the evening, with an interval of at least 8 hours between successive doses during the treatment phase.

Subjects were eligible for enrollment in the study if the following criteria were met at the start of the wash-out phase: a diagnosis of MDD based on the diagnostic criteria of the Diagnostic and Statistical Manual of Mental Disorders, 4th edition, text revision (DSM-IV-TR); ${ }^{6}$ a total score of $\geq 20$ on the Interactive Voice Response (IVR $)^{7-10}$-based Hamilton Rating Scale for Depression (HAM-D; 17 items); ${ }^{11}$ a total score of $\geq 25$ on the Inventory of Depressive Symptomatology-Self Report (IDS-SR); ; ${ }^{12,13}$ a score of $\geq 1$ on at least four of five items on the five-item subscale of the IDS-SR (items 19, 20, 21, 22, and 30), and a total score of $\geq 7$ on the five-item subscale of the IDS-SR; a Clinical
Global Impression Severity of Illness (CGI-SI) score of $\geq 4$ ("Moderately ill" or much worse); a current major depressive episode duration of $\geq 8$ weeks and $<24$ months; and aged $\geq 18$ and $<65$ years.

Subjects meeting any of the following criteria could not be enrolled in the study: past history of seizure or seizure disorder; more than a single febrile seizure in infancy; cerebral tumor; head/brain injury (traumatic); a history or current diagnosis of anorexia nervosa or bulimia; a primary DSM-IV diagnosis of, or received treatment for, panic disorder; obsessive compulsive disorder; post-traumatic stress disorder or acute stress disorder 12 months before the start of the run-in phase; a DSM-IV diagnosis of schizophrenia, or other psychotic disorder(s), including bipolar disorder; a history of, or currently has, manic episode(s); poses a current serious suicidal risk or has made a suicide attempt within the past 6 months; pregnant, possibly pregnant, lactating women, or females who want to become pregnant during the study. Concomitant use of drugs considered to have influence on efficacy evaluation was prohibited during the study periods.

Subjects were admitted to proceed to the treatment phase if the following criteria were met at the start of the treatment phase (visit 2, week 0): a total score of $\geq 20$ of the IVR-based HAM-D (17 items); IVR-based HAM-D (17 items) total score did not increase or decrease by $\geq 25 \%$ between visit 1 and visit 2; a total score of $\geq 25$ on the IDS-SR; a score of $\geq 1$ on at least four of five items on the five-item subscale of the IDS-SR and a total score of $\geq 7$ on the five-item subscale of the IDS-SR; and a CGI-SI score of $\geq 4$ ("Moderately ill" or much worse).

In order to avoid rater bias in the primary rating scale of the MADRS among investigators, we provided hands-on training courses to all study investigators prior to involvement in this study.

Investigators were required to use the MINI (Mini International Neuropsychiatric Interview) for the diagnosis of MDD, and this was explicitly defined in the study protocol.

The study protocol was prepared according to the International Conference on Harmonisation - Good Clinical Practice guidelines ${ }^{14}$ and reviewed and approved by the institutional review boards of the participating institutions prior to study initiation. The investigator ensured potential subjects of the study were fully informed, including the provision of written information; written informed consent was obtained from all patients prior to participation in the study. This study 
was conducted in accordance with the guiding principles of the Declaration of Helsinki (2008).

\section{Statistical methods}

\section{Sample size considerations}

The mean differences to be detected between each of the bupropion SR dose groups and the placebo group was set at 2.7, and a standard deviation of 9.0 for each group was assumed for the changes from baseline in MADRS total score at week 8. A total of 564 subjects (188 subjects per group) were required to test the statistical hypothesis of this study, with $80 \%$ power in comparison of each of the bupropion SR dose group versus placebo group, with multiplicity adjustment using Dunnett's step-down closed testing procedure.

\section{Efficacy measures}

\section{Primary endpoint}

The primary endpoint of this study was the change from baseline in MADRS total score at week 8 . The primary comparisons between the BUP150 and placebo groups, and between the BUP300 and placebo groups were made using the intent-to-treat (ITT) data set with missing values imputed using the last observation carried forward (LOCF) method. The mean change from baseline in MADRS total score at week 8 was compared between each of the bupropion SR dose groups and the placebo group using an analysis of covariance (ANCOVA) with Dunnett's step-down procedure to control inflation in the family-wise type I error rate. The procedure started with the comparison of primary interest and then stepping-down to 'the least significant' comparison only when the previous comparison was statistically significant. The ANCOVA model included region (country) and baseline value of MADRS total score (week 0) as covariates.

\section{Secondary endpoints}

For rating scales, observed scores and their change from baseline scores were summarized by treatment at each scheduled assessment point. The mean differences between each of the bupropion SR dose groups and the placebo group were estimated, along with 95\% confidence intervals, and the statistical comparison was made using ANCOVA. The ANCOVA models included region and baseline value as covariates.

\section{Safety measures}

Serious adverse events (SAEs) were recorded from the date the informed consent was obtained to the last follow-up contact, and other adverse events (AEs) were documented from the start of the investigational product to the end of the follow-up period. AEs, including SAEs, were recorded with non-leading questions. AEs leading to discontinuation of investigational product or withdrawal from the study were also documented.

Clinical laboratory data, vital sign data, and 12-lead electrocardiograph (ECG) findings were summarized at each scheduled assessment point.

\section{Results \\ Demography}

A total of 569 subjects were randomized to one of the three treatment groups: 187 in the placebo group, 190 in the BUP150 group, and 192 in the BUP300 group. Four subjects (one in the placebo group, three in the BUP300 group) who had not taken any investigational product were excluded from the safety-analysis population (SP). Among the SP, one subject in the BUP300 group for whom no efficacy observations had been recorded was excluded from the ITT analysis.

In the ITT analysis, there was no imbalance observed between treatment groups for any demographic and baseline factors (Table 1).

On subgroup analysis between the countries, the mean ages of the subjects were slightly higher in Korean subjects: $38.7,42.6$, and 42.3 years in the placebo, BUP150, and BUP300 groups, respectively, and 35.4, 36.1, and 36.8 years, respectively, in Japanese subjects. The proportion of female subjects was higher in Korean subjects: 70\%, 72\%, and 75\% in the placebo, BUP150, and BUP300 groups, respectively, and $50 \%, 47 \%$, and 51\%, respectively, in Japanese subjects. Except for the mean age and the ratio of males to females, subject demographic and baseline factors showed similar profiles in both countries.

\section{Efficacy results}

The adjusted mean (standard error $[\mathrm{SE}]$ ) of the change from baseline and comparison between each treatment group of bupropion SR over placebo are shown in Table 2. The mean MADRS total score decreased from baseline in all groups; however, there was no statistically significant difference between the placebo group and each treatment group. Since the first comparison (placebo versus [vs] BUP150) failed to show significance, the second comparison (placebo vs BUP300) was not performed, in order to control type I error rate.

Results of the secondary efficacy variables were consistent with results of the primary analysis. 
Table I Summary of pretreatment demographic and baseline characteristics (intent-to-treat population)

\begin{tabular}{|c|c|c|c|c|}
\hline & $\begin{array}{l}\text { Placebo } \\
(n=186)\end{array}$ & $\begin{array}{l}\text { BUP I } 50 \\
(n=190)\end{array}$ & $\begin{array}{l}\text { BUP300 } \\
(n=188)\end{array}$ & $\begin{array}{l}\text { Total } \\
(n=564)\end{array}$ \\
\hline \multicolumn{5}{|l|}{ Country (n) } \\
\hline Japan & 149 & 154 & 148 & 451 \\
\hline South Korea & 37 & 36 & 40 & 113 \\
\hline \multicolumn{5}{|l|}{ Age (years) } \\
\hline Mean & 37.9 & 36.0 & 37.5 & 37.1 \\
\hline SD & 11.09 & 10.42 & 10.96 & 10.84 \\
\hline Median & 37.0 & 35.0 & 36.0 & 36.0 \\
\hline Min & 20 & 18 & 21 & 18 \\
\hline $\operatorname{Max}$ & 63 & 64 & 64 & 64 \\
\hline \multicolumn{5}{|l|}{ Sex } \\
\hline Female & 101 (54\%) & 98 (52\%) & $105(56 \%)$ & 304 (54\%) \\
\hline Male & $85(46 \%)$ & 92 (48\%) & $83(44 \%)$ & $26 \mathrm{I}(46 \%)$ \\
\hline \multicolumn{5}{|l|}{ Weight (kg) } \\
\hline Mean & 60.19 & 61.01 & 62.00 & 61.07 \\
\hline SD & 11.678 & 12.793 & 13.164 & 12.564 \\
\hline Median & 58.45 & 59.00 & 59.95 & 59.00 \\
\hline Min & 37.5 & 39.0 & 38.8 & 37.5 \\
\hline Max & 95.2 & 107.4 & 102.0 & 107.4 \\
\hline \multicolumn{5}{|c|}{ DSM-IV-TR diagnosis } \\
\hline 296.20 & I (<I\%) & 0 & $\mathrm{I}(<\mathrm{I} \%)$ & $2(<1 \%)$ \\
\hline 296.21 & 0 & $\mathrm{I}(<\mathrm{l} \%)$ & 0 & $\mathrm{I}(<\mathrm{I} \%)$ \\
\hline 296.22 & $83(45 \%)$ & $84(44 \%)$ & 71 (38\%) & $238(42 \%)$ \\
\hline 296.23 & $14(8 \%)$ & $15(8 \%)$ & II (6\%) & $40(7 \%)$ \\
\hline 296.31 & 0 & $2(1 \%)$ & 0 & $2(<1 \%)$ \\
\hline 296.32 & 81 (44\%) & $76(40 \%)$ & 77 (4I\%) & $234(41 \%)$ \\
\hline 296.33 & $7(4 \%)$ & $12(6 \%)$ & $28(15 \%)$ & $47(8 \%)$ \\
\hline \multicolumn{5}{|c|}{ Number of previous depressive episodes (not including current episode) } \\
\hline 0 & $98(53 \%)$ & $100(53 \%)$ & $83(44 \%)$ & $28 \mathrm{I}(50 \%)$ \\
\hline 1 & $64(34 \%)$ & $56(29 \%)$ & $71(38 \%)$ & $191(34 \%)$ \\
\hline 2 & $12(6 \%)$ & $22(12 \%)$ & $23(12 \%)$ & $57(10 \%)$ \\
\hline 3 & $8(4 \%)$ & $6(3 \%)$ & $7(4 \%)$ & $21(4 \%)$ \\
\hline 4 or more & $4(2 \%)$ & $6(3 \%)$ & $4(2 \%)$ & $14(2 \%)$ \\
\hline \multicolumn{5}{|c|}{ Duration of current major depressive episode (weeks) } \\
\hline Mean & 26.8 & 26.5 & 28.7 & 27.3 \\
\hline SD & 19.44 & 19.18 & 22.27 & 20.33 \\
\hline Median & 21.0 & 19.5 & 22.0 & 21.0 \\
\hline Min & 8 & 8 & 8 & 8 \\
\hline Max & 100 & 98 & 101 & 101 \\
\hline
\end{tabular}

Notes: DSM-IV TR diagnosis code. 296.2: Major depressive disorder, single episode; 296.3: Major depressive disorder, recurrent.

Abbreviations: BUP150, bupropion SR $150 \mathrm{mg} /$ day (once daily); BUP300, bupropion SR $150 \mathrm{mg} /$ day (twice daily); DSM-IV-TR, Diagnostic and Statistical Manual of Mental Disorders, 4th edition, text revision; SD, standard deviation; SR, sustained release; Min, minimum; Max, maximum; $\mathrm{n}$, number of subjects.
MADRS total scores, from week 1 through week 8 , decreased over time in the bupropion SR treatment groups, but none of the reductions were significantly different from placebo (Table 3). IDS-SR total scores decreased over time in the bupropion SR treatment groups, but none of the reductions were significantly different from placebo (Table 4).

These efficacy results were consistent between the two countries participating in this study: Japan and Korea (Table 5).

Energy-related MADRS items (item 1, apparent sadness; item 2, reported sadness; item 6, concentration difficulties; item 7, lassitude; and item 8, inability to feel) were extracted to provide for the stratification analysis. The mean change from baseline in each item score of the five MADRS items decreased over time in every group. However, at any assessment point, there was no statistically significant difference in any item score between the placebo and each of the bupropion SR dose groups $(P=0.054$ to 0.922$)$.

MADRS responders were defined as "subject with $\mathrm{a} \geq 50 \%$ reduction from baseline in the MADRS total score at week 8 " and the MADRS remitters were defined as "subject with $\leq 11$ MADRS total score at week 8 ". There was no statistically significant difference between the placebo group and each of the bupropion SR dose groups in relation to MADRS responders and remitters (Table 6).

Post hoc subgroup analysis suggested that the patients who were diagnosed as "severe MDD" according to the DSM-IV-TR at baseline tended to show higher responses in bupropion treatment groups over placebo when comparing change in MADRS total scores from baseline. In this post hoc protocol compatible population analysis, without missing values complemented, the mean difference versus placebo in MADRS total scores at week 8 were -4.9 (SE 3.45) and -2.6 (SE 3.33) in the BUP150 and BUP300 groups, respectively. However, there was no statistical significance observed because of the limited number of subjects involved in the subgroup analysis.

Table 2 Change from baseline in MADRS total score at week 8 (ITT-LOCF)

\begin{tabular}{|c|c|c|c|c|c|c|c|c|}
\hline \multirow{2}{*}{$\begin{array}{l}\text { Treatment } \\
\text { group }\end{array}$} & \multirow[t]{2}{*}{$\mathbf{n}$} & \multirow{2}{*}{$\begin{array}{l}\text { Adjusted } \\
\text { mean }\end{array}$} & \multirow[t]{2}{*}{ SE } & \multicolumn{5}{|c|}{ Difference vs placebo } \\
\hline & & & & Mean & SE & $\begin{array}{l}\text { Order } \\
\text { of test }\end{array}$ & $\begin{array}{l}\text { Adjusted } \\
95 \% \mathrm{Cl}\end{array}$ & $\begin{array}{l}\text { Adjustec } \\
\text { P-value }\end{array}$ \\
\hline Placebo & 186 & -13.9 & 0.77 & - & - & - & - & - \\
\hline BUPI50 & 190 & -14.4 & 0.77 & -0.5 & 1.00 & I & $-2.7,1.7$ & 0.853 \\
\hline BUP300 & 188 & -12.9 & 0.76 & 1.0 & 1.00 & 2 & $N / A$ & $N / A$ \\
\hline
\end{tabular}

Notes: $\mathrm{Cls}$ and the adjusted $P$-values were based on Dunnett's step-down procedure. The statistical model included treatment group, region (country), and baseline value as explanatory variables. Since the first comparison (placebo vs BUPI50) failed to show significance, the second comparison (placebo vs BUP300) was not performed, in order to control type I error rate.

Abbreviations: BUPI50, bupropion SR I50 mg/day (once daily); BUP300, bupropion SR 150 mg/day (twice daily); Cl, confidence interval; ITT-LOCF, intent-to-treat, last observation carried forward; MADRS, Montgomery-Åsberg Depression Rating Scale; N/A, not applicable; SE, standard error; SR, sustained release; n, number; vs, versus. 
Table 3 Comparison of change from baseline in MADRS total scores (ITT-LOCF)

\begin{tabular}{|c|c|c|c|c|c|c|c|}
\hline \multirow[t]{2}{*}{ Visit } & \multirow{2}{*}{$\begin{array}{l}\text { Treatment } \\
\text { group }\end{array}$} & \multirow[t]{2}{*}{$\mathbf{n}$} & \multirow{2}{*}{$\begin{array}{l}\text { Adjusted } \\
\text { mean }\end{array}$} & \multirow[t]{2}{*}{ SE } & \multicolumn{3}{|c|}{ Difference vs placebo } \\
\hline & & & & & Mean & SE & $95 \% \mathrm{Cl}$ \\
\hline \multirow[t]{3}{*}{ Week I } & Placebo & 186 & -3.4 & 0.38 & - & - & - \\
\hline & BUPI50 & 190 & -2.9 & 0.38 & 0.6 & 0.48 & $-0.4,1.5$ \\
\hline & BUP300 & 188 & -2.7 & 0.38 & 0.7 & 0.49 & $-0.2,1.7$ \\
\hline \multirow[t]{3}{*}{ Week 2} & Placebo & 186 & -6.2 & 0.47 & - & - & - \\
\hline & BUPI50 & 190 & -5.0 & 0.47 & 1.2 & 0.61 & $0.0,2.4$ \\
\hline & BUP300 & 188 & -5.0 & 0.47 & 1.2 & 0.61 & $-0.1,2.4$ \\
\hline \multirow[t]{3}{*}{ Week 4} & Placebo & 186 & -9.2 & 0.61 & - & - & - \\
\hline & BUPI50 & 190 & -8.3 & 0.60 & 0.9 & 0.78 & $-0.7,2.4$ \\
\hline & BUP300 & 188 & -8.2 & 0.60 & 1.0 & 0.78 & $-0.6,2.5$ \\
\hline \multirow[t]{3}{*}{ Week 6} & Placebo & 186 & -11.8 & 0.70 & - & - & - \\
\hline & BUPI50 & 190 & -11.7 & 0.70 & 0.1 & 0.90 & $-1.7,1.8$ \\
\hline & BUP300 & 188 & -11.1 & 0.69 & 0.7 & 0.90 & $-I . I, 2.4$ \\
\hline \multirow[t]{3}{*}{ Week 8} & Placebo & 186 & -13.9 & 0.77 & - & - & - \\
\hline & BUPI50 & 190 & -14.4 & 0.77 & -0.5 & 1.00 & $-2.4,1.5$ \\
\hline & BUP300 & 188 & -12.9 & 0.76 & 1.0 & 1.00 & $-1.0,3.0$ \\
\hline
\end{tabular}

Abbreviations: BUPI50, bupropion SR 150 mg/day (once daily); BUP300, bupropion SR 150 mg/day (twice daily); Cl, confidence interval; ITT-LOCF, intent-to-treat, last observation carried forward; MADRS, Montgomery-Åsberg Depression Rating Scale; SE, standard error; SR, sustained release; n, number; vs, versus.

\section{Safety results}

The percentage of subjects who reported at least one AE during the treatment phase was slightly higher in the BUP300 group (65\%) than in the placebo (55\%) and BUP150 (56\%) groups. The most frequent AEs, which were reported in at least $5 \%$ of the subjects in any of the treatment groups, were nasopharyngitis, dry mouth, headache, nausea, constipation, tremor, and insomnia (Table 7). In all treatment groups, the majority of AEs reported were considered mild or moderate in intensity. There were no seizures or change to manic state observed in any of the treatment groups. Non-fatal SAEs were reported by $1 \%$ of subjects in the placebo group, $1 \%$ of subjects in the BUP150 group, and less than $1 \%$ of subjects in the BUP300 group. All of those SAEs resolved. The number of subjects reporting AEs leading to discontinuation of investigational product or withdrawal from the study was low and similar across treatment groups: four (2\%) subjects

Table 4 Comparison of change from baseline in IDS-SR total scores (ITT-LOCF)

\begin{tabular}{|c|c|c|c|c|c|c|c|}
\hline \multirow[t]{2}{*}{ Visit } & \multirow{2}{*}{$\begin{array}{l}\text { Treatment } \\
\text { group }\end{array}$} & \multirow[t]{2}{*}{$\mathbf{n}$} & \multirow{2}{*}{$\begin{array}{l}\text { Adjusted } \\
\text { mean }\end{array}$} & \multirow[t]{2}{*}{ SE } & \multicolumn{3}{|c|}{ Difference vs placebo } \\
\hline & & & & & Mean & SE & $95 \% \mathrm{Cl}$ \\
\hline \multirow[t]{3}{*}{ Week I } & Placebo & 186 & -4.8 & 0.57 & - & - & - \\
\hline & BUPI50 & 190 & -4.2 & 0.57 & 0.6 & 0.73 & $-0.8,2.1$ \\
\hline & BUP300 & 188 & -3.9 & 0.57 & 0.9 & 0.73 & $-0.6,2.3$ \\
\hline \multirow[t]{3}{*}{ Week 2} & Placebo & 186 & -6.9 & 0.68 & - & - & - \\
\hline & BUPI50 & 190 & -6.7 & 0.68 & 0.2 & 0.88 & $-1.5,1.9$ \\
\hline & BUP300 & 188 & -5.8 & 0.68 & I.I & 0.88 & $-0.7,2.8$ \\
\hline \multirow[t]{3}{*}{ Week 4} & Placebo & 186 & -9.7 & 0.82 & - & - & - \\
\hline & BUPI50 & 190 & -9.3 & 0.82 & 0.4 & 1.06 & $-1.7,2.5$ \\
\hline & BUP300 & 188 & -8.2 & 0.81 & 1.5 & 1.06 & $-0.6,3.6$ \\
\hline \multirow[t]{3}{*}{ Week 6} & Placebo & 186 & -11.5 & 0.88 & - & - & - \\
\hline & BUPI50 & 190 & -12.3 & 0.88 & -0.8 & 1.14 & $-3.0,1.4$ \\
\hline & BUP300 & 188 & -10.9 & 0.87 & 0.5 & 1.14 & $-1.7,2.8$ \\
\hline \multirow[t]{3}{*}{ Week 8} & Placebo & 186 & -13.6 & 0.95 & - & - & - \\
\hline & BUPI50 & 190 & -14.5 & 0.95 & -0.9 & 1.23 & $-3.3,1.5$ \\
\hline & BUP300 & 188 & -12.6 & 0.94 & 1.0 & 1.24 & $-1.5,3.4$ \\
\hline
\end{tabular}

Notes: Adjusted means, differences, SEs, Cls, and P-values were based on analyses of covariance. For overall comparisons, statistical models included treatment, baseline value, and region as explanatory variables. For comparisons by region in Japan and Korea, statistical models included treatment and baseline value as explanatory variables. Abbreviations: BUPI50, bupropion SR I50 mg/day (once daily); BUP300, bupropion SR I50 mg/day (twice daily); Cl, confidence interval; IDS-SR, Inventory of Depressive Symptomatology-Self Report; ITT-LOCF, intent-to-treat, last observation carried forward; SE, standard error; SR, sustained release; n, number; vs, versus. 
Table 5 Change from baseline in MADRS total score at week 8 stratified by region (ITT-LOCF)

\begin{tabular}{|c|c|c|c|c|c|c|}
\hline \multirow{2}{*}{$\begin{array}{l}\text { Treatment } \\
\text { group }\end{array}$} & \multicolumn{3}{|c|}{ Japan } & \multicolumn{3}{|c|}{ South Korea } \\
\hline & $\bar{n}$ & $\begin{array}{l}\text { Mean } \\
\text { (SD) }\end{array}$ & $\begin{array}{l}\text { Min, } \\
\text { Max }\end{array}$ & $\bar{n}$ & $\begin{array}{l}\text { Mean } \\
(\mathrm{SD})\end{array}$ & $\begin{array}{l}\text { Min, } \\
\text { Max }\end{array}$ \\
\hline Placebo & 149 & $\begin{array}{l}-14.9 \\
(10.38)\end{array}$ & $-43,10$ & 37 & $\begin{array}{l}-12.1 \\
(10.06)\end{array}$ & $-33,4$ \\
\hline BUPI50 & 154 & $\begin{array}{l}-15.2 \\
(10.14)\end{array}$ & $-42,5$ & 36 & $\begin{array}{l}-12.8 \\
(8.86)\end{array}$ & $-33,1$ \\
\hline BUP300 & 148 & $\begin{array}{l}-13.9 \\
(10.64)\end{array}$ & $-42,13$ & 40 & $\begin{array}{l}-11.8 \\
(9.15)\end{array}$ & $-28,4$ \\
\hline
\end{tabular}

Abbreviations: BUPI50, bupropion SR $150 \mathrm{mg} /$ day (once daily); BUP300, bupropion SR $150 \mathrm{mg} /$ day (twice daily); ITT-LOCF, intent-to-treat, last observation carried forward; MADRS, Montgomery-Åsberg Depression Rating Scale; SD, standard deviation; SR, sustained release; $n$, number; Min, minimum; Max, maximum.

in the placebo group, $12(6 \%)$ in the BUP150 group, and nine $(5 \%)$ in the BUP300 group.

Throughout the study period, there was no clinically meaningful change in any laboratory parameters or in any vital sign values.

\section{Discussion}

A total of 569 subjects were enrolled in this Asian multinational study of bupropion SR for the treatment of MDD. The group proceeding to the treatment phase included 454 Japanese subjects and 115 Korean subjects. Although the mean ages of the Korean subject groups were slightly higher than those of the Japanese subject groups, and the proportion of women enrolled in the Korean subject groups was higher than that of the Japanese subject groups, no imbalance was noted in any demographic and baseline factors and there was no concern regarding the comparability of the treatment groups.

Use of the IVR HAM-D assessment at the screening phase succeeded in reducing the enrollment of subjects with mild depression, which is often reported as an important factor in causing high placebo response rates in clinical trials of antidepressants. ${ }^{15}$ The average MADRS total scores at baseline (week 0) were 31.9, 31.8, and 32.1 in the placebo, BUP150, and BUP300 groups, respectively. MADRS was employed for the primary assessment of depression in the treatment phase rather than the continuous use of the HAM-D, carrying over from the screening phase, in order to suppress potential rater's bias.

There was no statistically significant difference between the placebo and each of the bupropion SR dose groups in the primary efficacy endpoint (change from baseline in MADRS total score at week 8). It has been reported that bupropion appears to be potentially beneficial in the treatment of MDDrelated fatigue. ${ }^{16}$ However, no difference was shown between the placebo and each of the bupropion SR dose groups at any treatment visit in the mean change from baseline for MADRS item 7 (lassitude), which can be assumed to be a fatigue-related rating score. Nor did the secondary analysis and the other subgroup analysis provide a statistical significance in efficacy.

The most probable reason for our inability to show superiority of bupropion would be the so-called 'placebo effect'. Taking into account the fact that the placebo effect has been increasing over time in clinical studies of bupropion, ${ }^{17}$ a high placebo effect may have affected the assessment of the efficacy of bupropion SR, although analyses for various factors performed did not provide any conclusive evidence. The authors were not able to detect a relationship between individual study settings and degree of placebo response. Further examination would be necessary to identify the potential factors inflating the placebo effect.

AEs were reported in 106 (56\%) and 123 (65\%) subjects in the BUP150 and BUP300 groups, respectively, and $103(55 \%)$ subjects in the placebo group, which indicated that there was no substantial difference in the incidence of AEs between treatment groups. The AEs commonly reported in any treatment groups included nasopharyngitis, dry mouth, headache, nausea, constipation, and insomnia, which were similar to those reported in a meta-analysis of

Table 6 Summary of treatment difference for MADRS responders and remitters at week 8 (ITT-LOCF)

\begin{tabular}{|c|c|c|c|c|c|c|c|}
\hline \multirow{2}{*}{$\begin{array}{l}\text { Treatment } \\
\text { group }\end{array}$} & \multirow[t]{2}{*}{$\mathbf{n}$} & \multicolumn{3}{|c|}{ MADRS responder } & \multicolumn{3}{|c|}{ MADRS remitter } \\
\hline & & $\begin{array}{l}\text { Responders } \\
\text { (\%) }\end{array}$ & $\begin{array}{l}\text { Difference (\%) } \\
\text { vs placebo }\end{array}$ & $\begin{array}{l}95 \% \mathrm{Cl} \text { for } \\
\text { difference (\%) }\end{array}$ & $\begin{array}{l}\text { Remitters } \\
\text { (\%) }\end{array}$ & $\begin{array}{l}\text { Difference (\%) } \\
\text { vs placebo }\end{array}$ & $\begin{array}{l}95 \% \mathrm{Cl} \text { for } \\
\text { difference (\%) }\end{array}$ \\
\hline Placebo & 186 & $86(46.2)$ & - & - & $53(28.5)$ & - & - \\
\hline BUPI50 & 190 & $98(51.6)$ & -5.3 & $-15.4,4.7$ & $60(31.6)$ & -3.1 & $-12.3,6.2$ \\
\hline BUP300 & 188 & $82(43.6)$ & 2.6 & $-7.5,12.7$ & $56(29.8)$ & -1.3 & $-10.5,7.9$ \\
\hline
\end{tabular}

Notes: An MADRS responder is defined as a subject with a $\geq 50 \%$ reduction from baseline in the MADRS total score at week 8 . MADRS remitter is defined as a subject with $\leq$ II MADRS total score at week 8 .

Abbreviations: BUPI50, bupropion SR 150 mg/day (once daily); BUP300, bupropion SR I50 mg/day (twice daily); Cl, confidence interval; ITT-LOCF, intent-to-treat, last observation carried forward; MADRS, Montgomery-Åsberg Depression Rating Scale; SR, sustained release; vs, versus; $n$, number. 
Table 7 Adverse events reported in at least $5 \%$ of the subjects in any treatment group (safety-analysis population)

\begin{tabular}{|c|c|c|c|}
\hline System organ class & Placebo & BUPI50 & BUP300 \\
\hline Preferred term & $(n=186)$ & $(n=190)$ & $(n=189)$ \\
\hline $\mathrm{n}(\%)$ of subjects with any $\mathrm{AE}$ & $103(55)$ & $106(56)$ & $123(65)$ \\
\hline Gastrointestinal disorders & $39(2 I)$ & $47(25)$ & $63(33)$ \\
\hline Dry mouth & $8(4)$ & $12(6)$ & $28(15)$ \\
\hline Nausea & $15(8)$ & $13(7)$ & $16(8)$ \\
\hline Constipation & $3(2)$ & $8(4)$ & II (6) \\
\hline Infections and infestations & $42(23)$ & $28(15)$ & $35(19)$ \\
\hline Nasopharyngitis & $35(19)$ & $26(14)$ & $29(15)$ \\
\hline Nervous system disorders & $29(16)$ & $29(15)$ & $37(20)$ \\
\hline Headache & $13(7)$ & $16(8)$ & $19(10)$ \\
\hline Tremor & 0 & $4(2)$ & $9(5)$ \\
\hline Psychiatric disorders & $14(8)$ & $9(5)$ & $18(10)$ \\
\hline Insomnia & $6(3)$ & 0 & $10(5)$ \\
\hline
\end{tabular}

Abbreviations: AE, adverse event; BUPI50, bupropion SR $150 \mathrm{mg} /$ day (once daily); BUP300, bupropion SR 150 mg/day (twice daily); n, number.

overseas double-blind comparative studies. ${ }^{18}$ There were no particular findings in subjects treated with bupropion $\mathrm{SR}$ in regard to sexual dysfunction, weight change, and withdrawal syndrome, which are frequently recognized as clinical concerns associated with SSRIs, which are widely used for the treatment of depression. ${ }^{19-21}$ There were no seizures in any of the treatment groups. SAEs were reported in two subjects in the placebo group, two subjects in the BUP150 group, and one subject in the BUP300 group. All of these SAEs resolved. The incidence of suicidal ideation based on the Columbia Suicide Severity Rating Scale 22 was similar between treatment groups, and there were no reports of completed suicide. There were no clinically significant findings in clinical laboratory parameters and vital signs.

These results concerning AEs, and the other safety findings in terms of their type, severity, and incidence, indicate a favorable safety profile for bupropion SR.

\section{Conclusion}

This study was carried out as the first Asian collaboration study of bupropion SR for the treatment of the patients with MDD.

The mean change from baseline in MADRS total score at week 8 (ITT-LOCF), the primary efficacy endpoint, decreased in all of the three treatment groups (placebo, BUP150, and BUP300); however, there was no statistically significant difference between the placebo group and each of the bupropion SR dose groups. Similar results were generally obtained for all of the secondary efficacy endpoints.

There was no substantial difference in the type, severity, and incidence of AEs between the bupropion SR dose groups and the placebo group, which indicated a favorable safety profile for bupropion SR.

Although this study did not demonstrate superiority of bupropion SR over placebo, due to a high placebo response, the drug was well tolerated and no new safety concerns were identified in this Asian population.

\section{Acknowledgments and disclosure}

This study was funded and conducted by GlaxoSmithKline. All listed authors meet the criteria for authorship set forth by the International Committee for Medical Journal Editors. Yoshifumi Koshino received research funding from GlaxoSmithKline as a medical expert and sponsor's responsible medical officer. Won-Myong Bahk and Hideaki Sakai received grant support from GlaxoSmithKline as investigators. Takayuki Kobayashi is a full-time employee of GlaxoSmithKline. The authors deeply appreciate all the investigators participating in the study. The authors report no other conflicts of interest in this work.

\section{References}

1. Fava M, Rush AJ, Thase ME, et al. 15 years of clinical experience with bupropion $\mathrm{HCl}$ : from bupropion to bupropion $\mathrm{SR}$ to bupropion $\mathrm{XL}$. Prim Care Companion J Clin Psychiatry. 2005;7:106-113.

2. Cipriani A, Furukawa TA, Salanti G, et al. Comparative efficacy and acceptability of 12 new- generation antidepressants: a multipletreatments meta-analysis. Lancet. 2009;373(9665):746-758.

3. Coleman CC, King BR, Bolden-Watson C, et al. A placebo-controlled comparison of the effects on sexual functioning of bupropion sustained release and fluoxetine. Clin Ther. 2001;23:1040-1058.

4. Jefferson JW, Rush AJ, Nelson JC, et al. Extended-release bupropion for patients with major depressive disorder presenting with symptoms of reducing energy, pleasure, and interest: findings from a randomized, double-blind, placebo-controlled study. J Clin Psychiatry. 2006;67: 865-873.

5. Montgomery SA, Åsberg M. A new depression rating scale designed to be sensitive to change. Br J Psychiatry. 1979;134:382-389.

6. American Psychiatric Association. Diagnostic and Statistical Manual of Mental Disorders. 4th ed. Text revision. Washington, DC; American Psychiatric Association; 2000.

7. Mundt JC, Greist JH, Jefferson JW, et al. Is it easier to find what you are looking for if you think you know what it looks like? J Clin Psychopharmacol. 2007;27:121-125.

8. Mundt JC, Snyder PJ, Cannizzaro MS, Chappie K, Geralts DS. Voice acoustic measures of depression severity and treatment response collected via interactive voice response (IVR) technology. J Neurolinguistics. 2007;20:50-64.

9. Kobak KA, Reynolds WM, Rosenfeld R, Greist JH. Development and validation of a computer-administered version of the Hamilton Depression Rating Scale. Psychol Assess. 1990;2:56-63.

10. Kunigi H, Koga N, Hashikura M, et al. Validation of computeradministered clinical rating scale: Hamilton Depression Rating Scale assessment with Interactive Voice Response technology - Japanese version. Psychiatry Clin Neurosci. 2013;67:253-258.

11. Hamilton M. A rating scale for depression. J Neurol Neurosurg Psychiatry. 1960;23:56-62.

12. Rash AJ, Gullion CM, Basco MR, Jarrett RB, Trivedi MH. The Inventory of Depressive Symptomatology (IDS): psychometric properties. Psychol Med. 1996;26:477-486. 
13. Rush AJ, Carmody T, Reimitz PE. The Inventory of Depressive Symptomatology (IDS): Clinician (IDS-C) and Self-Report (IDS-SR) ratings of depressive symptoms. Int J Methods Psychiatr Res. 2000;9: 45-59.

14. International Conference on Harmonisation of technical requirements for registration of pharmaceuticals for human use. ICH Harmonized Tripartite Guideline: guideline for good clinical practice. J Postgrad Med. 2001;47:199-203.

15. Posternak MA, Zimmerman M, Keitner GI, Miller IW. A reevaluation of the exclusion criteria used in antidepressant efficacy trials. Am J Psychiatry. 2002;159:191-200.

16. Pae CU, Lim HK, Han C, et al. Fatigue as a core symptom in major depressive disorder: overview and the role of bupropion. Expert Rev Neurother. 2007; 7:1251-1263.

17. Lieberman JA, Greenhouse J, Hamer RM, et al. Comparing the effects of antidepressants: consensus guidelines for evaluating quantitative reviews of antidepressant efficacy. Neuropsychopharmacology. 2005;30:445-460
18. Thase ME, Haight BR, Richard N, et al. Remission rates following antidepressant therapy with bupropion or selective serotonin reuptake inhibitors: a meta-analysis of original data from 7 randomized controlled trials. J Clin Psychiatry. 2005;66:974-981.

19. Clayton AH, Pradko JF, Croft HA, et al. Prevalence of sexual dysfunction among newer antidepressants. J Clin Psychiatry. 2002;63:357-366.

20. Serretti A, Mandelli L. Antidepressants and body weight: a comprehensive review and meta-analysis. J Clin Psychiatry. 2010;71:1259-1272.

21. Haddad PM. Antidepressant discontinuation syndromes. Drug Saf. 2001;24(3):183-197.

22. Columbia University Medical Center. Columbia-Suicide Severity Rating Scale (C-SSRS) [homepage on the internet]. Available from: http://www. cssrs.columbia.edu/. Accessed July 31, 2013.

\section{Publish your work in this journal}

Neuropsychiatric Disease and Treatment is an international, peerreviewed journal of clinical therapeutics and pharmacology focusing on concise rapid reporting of clinical or pre-clinical studies on a range of neuropsychiatric and neurological disorders. This journal is indexed on PubMed Central, the 'PsycINFO' database and CAS.
The manuscript management system is completely online and includes a very quick and fair peer-review system, which is all easy to use. Visit http://www.dovepress.com/testimonials.php to read real quotes from published authors. 